\title{
Suppression of Deflagration Flame Propagation of Methane-Air in Tube By Argon Gas and Explosion- Eliminating Chamber
}

Quan Wang ( $\nabla$ wqaust@163.com )

Anhui University of Science \& Technology

Xiaomeng Xu

Anhui University of Science \& Technology

Weida Chang

Northeastern University

Zhimin Li

Anhui University of Science \& Technology

Jun Zhang

Anhui University of Science \& Technology

Rui Li

Anhui University of Science \& Technology

\section{Research Article}

Keywords: explosion-eliminating chamber, argon, methane-air deflagration flame, suppression

Posted Date: September 29th, 2021

DOI: https://doi.org/10.21203/rs.3.rs-900922/v1

License: (c) (i) This work is licensed under a Creative Commons Attribution 4.0 International License.

Read Full License 


\title{
Suppression of deflagration flame propagation of methane-air in tube by argon gas and explosion-eliminating chamber
}

\author{
Quan Wang, ${ }^{\mathrm{a}, \mathrm{b}^{*}}$, Xiaomeng Xu' ${ }^{\mathrm{a}}$, Weida Chang ${ }^{\mathrm{d}}$, Zhimin Li ${ }^{\mathrm{c}}$, Jun Zhang ${ }^{\mathrm{a}}$, Rui Li ${ }^{\text {a** }}$ \\ a. School of Chemical Engineering, Anhui University of Science \& Technology, Huainan 232001, China \\ b. Engineering Laboratory of Explosive Materials and Technology of Anhui Province, Huainan 232001, China \\ c. School of Civil Engineering and Architecture, Anhui University of Science \& Technology, Huainan 232001, China \\ d. Fire \& Explosion Protection Laboratory, Northeastern University, Shenyang 110819, China
}




\begin{abstract}
To explore the inhibitory effect of argon gas and explosion-eliminating chamber on methane-air deflagration flame propagation in the tube, based on the $\Phi=120 \mathrm{~mm}, \mathrm{~L}=5.5 \mathrm{~m}$ stainless steel pipeline test system to measure methane-air deflagration flame structure, flame propagation speed, and deflagration pressure. The results show that: $10 \% \sim 30 \%$ argon is mixed into the methane-air premixed gas with different equivalent ratios. With the increase in the mixed argon content, the tensile distortion and instability of the flame front increase, and the average value of flame propagation speed decreases by $2.52 \% \sim 60.0 \%$. The first and second deflagration pressure peaks are reduced by about $13.1 \% \sim 62 \%$ and $17.7 \% \sim 86.5 \%$ respectively. The average value of the methane-air deflagration flame propagation velocity was reduced by $5.7 \% \sim 37.0 \%$ with the explosion-eliminating chamber laid at the nozzle. The second and third deflagration pressure peaks are reduced by about $10 \% \sim 30 \%$ and $50 \% \sim 90 \%$ respectively. The inhibitory effect of argon on the propagation of methane-air flame is considered better than the laying of the explosion-eliminating chamber under the experimental conditions.
\end{abstract}

Keywords: explosion-eliminating chamber; argon; methane-air deflagration flame; suppression

\title{
1 Introduction
}

Methane is widely used in daily production and life, but as a combustible gas, if it is not used properly, it may explode and cause serious harm. At present, the most commonly used method to prevent explosion hazards is to add certain inert medium in methane gas as flame retardant, such as nitrogen $\left(\mathrm{N}_{2}\right)$, carbon dioxide $\left(\mathrm{CO}_{2}\right)$ and water vapor $\left(\mathrm{H}_{2} \mathrm{O}\right)^{1-3}$. Inert medium can relatively reduce the volume fraction of combustible, so that it can be controlled beyond the explosion limit to achieve flame retardant effect. $\mathrm{Kondo}^{4,5}$ explored the effect of $\mathrm{CO}_{2}$ and $\mathrm{N}_{2}$ on the explosion limits of various combustible gases, and found that this effect can be explained by the extended Le Chatelier formula using a set of commonly used parameter values. M. Bundy ${ }^{6}$ studied the inhibition degree of inert gas $\mathrm{CO}_{2}, \mathrm{~N}_{2}$ and trifluoromethane

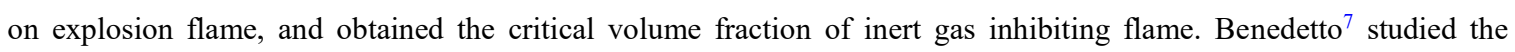
influence of $\mathrm{CO}_{2}$ content on the peak pressure, maximum pressure rise rate and laminar combustion rate, indicating that the main effect of $\mathrm{CO}_{2}$ is the effect on the specific heat of the mixture. When the $\mathrm{CO}_{2}$ content reduces the adiabatic flame temperature to about $1500 \mathrm{~K}$, the flame will be extinguished. It is shown that the main effect of $\mathrm{CO}_{2}$ is on the specific heat of the mixture. When the $\mathrm{CO}_{2}$ content decreases the adiabatic flame temperature down to about $1500 \mathrm{~K}$, the flame extinction occurs. C. V. Mashuga ${ }^{8}$ estimated the explosion limit of the mixture of methane / ethylene and nitrogen at the adiabatic flame temperature of $1200 \mathrm{~K}$, and there was a good agreement between the results and the experimental values. Y. N. Shebeko ${ }^{9}$ estimated the explosion limit of the ternary mixture of combustible and flame retardant at the adiabatic flame temperature of $1600 \mathrm{~K}$, and proposed a new method for calculating the explosion limit of the mixture. $\mathrm{M} \mathrm{Vidal}{ }^{10}$ calculated the adiabatic flame temperature by using SuperChems software to estimate the lower explosion limit of hydrocarbon / flame retardant mixture.

Besides, explosion venting as an effective disaster mitigation method has also been widely studied by scholars. Cooper ${ }^{11}$ explored the influence of vent area on the deflagration flame pressure peak. The first, third and fourth pressure peaks increase with the decrease of vent area, while the second pressure peak first increases and then decreases. Bauwens ${ }^{12}$ also found that the smaller vent area would lead to higher internal overpressure peak of the direct vented vessel. However, Ponizy $\mathrm{B}^{13}$ also show that the increase of vent area does not always lead to the decrease of overpressure peak. Ferrara ${ }^{14}$ explained this phenomenon as the result of the interaction between combustion rate and venting rate. Solberg ${ }^{15}$ found that the pressure of central ignition is the highest and the pressure rising speed is the 
fastest in the pipe with vent. Bradley ${ }^{16}$ thought that the most dangerous ignition position in the spherical vessel with vent is in the center of the vessel. Kasmani ${ }^{17}$ studied the explosion overpressure of methane air premixed gas in the cylindrical vessel with vent, and the tail ignition can cause more serious consequences. Alexiou ${ }^{18}$ found that when the pressure relief port is close to the ignition end, the explosion relief effect is the best, and when the pressure relief port is located in the middle of the pipeline, the explosion relief effect is the worst.

However, in large pipelines, argon is used as a diluent gas to suppress the propagation of methane-air deflagration flame and the related research on the explosion suppression device is relatively rare. Based on this, this article explores and compares the inhibitory effect of argon gas and explosion-eliminating chamber on methane-air deflagration flame propagation in the tube, according to the flame motion images, flame propagation speed, deflagration pressure, and other characteristic parameters.

\section{Experimental section}

\subsection{Materials}

Methane (99.99\%) and argon (99.999\%) were purchased from Hefei Henglong Electric Co., Ltd, (Hefei, P. R. China).

\subsection{Experimental system}

As shown in Fig. 1, the experimental system consists of a stainless steel flame acceleration pipeline, a gas distribution system, a detonation chamber, a high-speed camera, and a data acquisition system. The flame acceleration tube is a cylindrical tube of $\Phi=120 \mathrm{~mm}$ and $\mathrm{L}=5.5 \mathrm{~m}$, and an observation window of $40 \mathrm{~cm} \times 7 \mathrm{~cm}$ is laid at $130 \mathrm{~cm}$ from the ignition end of the tube. The ignition end of the pipeline is closed, and the open end is closed with 2 layers of polyethylene (PE) film with a thickness of $0.02 \mathrm{~mm}$.



Fig. 1 Experimental system 
A PCB pressure sensor (with a response time of fewer than $1 \mu \mathrm{s}$ ) is laid at $141 \mathrm{~cm}$ from the ignition end of the pipeline to monitor the deflagration pressure in the pipeline, while 6 flame sensors are laid at the pipeline with a horizontal distance from $226 \mathrm{~cm}$ to $476 \mathrm{~cm}$ (50 cm interval) from the ignition end to monitor the deflagration flame propagation velocity. The high-speed camera is used to monitor images of deflagration flames directly at the observation window at a shooting rate of $2000 \mathrm{fps}$ and save them in a computer.

As shown in Fig. 2, the explosion-eliminating chamber used in the experiment is an explosion suppression device with a volume of $1.53 \mathrm{~m}^{3}$, and three explosion suppression rings are installed at equal distances inside the tank. The explosion suppression ring described in this article is polyester fiber cotton mainly consisting of annular porous acoustic absorbent material.

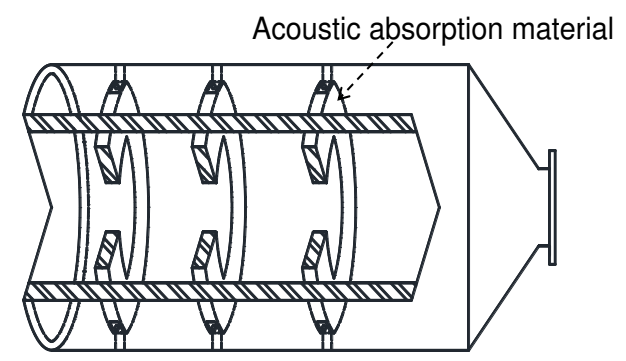

Fig. 2 Structure diagram of explosion-eliminating chamber

\subsection{Gas preparing scheme}

The pipeline is first evacuated to vacuum and then filled with the configured methane-air premixed gas to make the pipeline pressure return to $1 \mathrm{~atm}$ for the experiment. The formula for calculating the equivalent ratio of combustible gas is: In the formula: $\Phi$ is the combustible gas equivalent ratio, and $m$ is the gas mass. The gas composition required for the experiment is shown in Table 1. To ensure that the prepared gas is evenly mixed, it needs to be premixed in the air mixing tank for $3 \sim 5$ hours.

Table 1 Experimental gas preparing scheme for the suppression of methane-air deflagration flame in the explosion-eliminating chamber

\begin{tabular}{ccc}
\hline$\Phi$ & $\mathrm{CH}_{4}$ (vol. \%) & Air (vol. \%) \\
\hline 0.9 & 8.64 & 91.36 \\
1.0 & 9.50 & 90.5 \\
1.1 & 10.36 & 89.64 \\
\hline
\end{tabular}

When exploring the inhibitory effect of argon added on the deflagration flame of methane-air in the tube, there is no reaction between argon and the methane-air mixture, so the argon ratio can be calculated from: 


$$
\beta=\frac{V_{\text {dilution }}}{\mathrm{V}_{\text {dilution }}+\mathrm{V}_{\mathrm{CH}_{4}}+\mathrm{V}_{\mathrm{air}}} \times 100 \%
$$

Here, $V_{\text {dilution }}$ is the volume of inert gas, $V_{\text {air }}$ is the volume of air, and $V_{C H 4}$ is the volume of methane. The gas preparing scheme calculated is shown in Table 2 according to the above formula.

Table 2 The experimental gas distribution scheme of argon on the suppression of methane-air deflagration flame

\begin{tabular}{|c|c|c|c|c|c|c|c|c|c|c|}
\hline \multirow{2}{*}{$\beta$} & \multirow{2}{*}{${ }_{A r} / \%$} & \multicolumn{3}{|c|}{$\Phi=0.9$} & \multicolumn{3}{|c|}{$\Phi=1.0$} & \multicolumn{3}{|c|}{$\Phi=1.1$} \\
\hline & & $\mathrm{Ar} / \%$ & $\mathrm{CH}_{4} / \%$ & Air $/ \%$ & $\mathrm{Ar} / \%$ & $\mathrm{CH}_{4} / \%$ & Air $/ \%$ & $\mathrm{Ar} / \%$ & $\mathrm{CH}_{4} / \%$ & Air $/ \%$ \\
\hline & 0 & 0.00 & 8.64 & 91.36 & 0.00 & 9.50 & 90.50 & 0.00 & 10.36 & 89.64 \\
\hline & 10 & 10.00 & 7.78 & 82.22 & 10.00 & 8.55 & 81.45 & 10.00 & 9.31 & 80.69 \\
\hline & 20 & 20.00 & 6.91 & 73.09 & 20.00 & 7.60 & 72.40 & 20.00 & 8.29 & 71.71 \\
\hline & 30 & 30.00 & 6.05 & 63.95 & 30.00 & 6.65 & 63.35 & 30.00 & 7.25 & 62.75 \\
\hline
\end{tabular}

\section{Experimental results and discussion}

\subsection{Effect of argon on the flame propagation characteristics of methane-air deflagration}

\subsubsection{Effect of argon on deflagration flame profile}

Figures 3 to 5 are photos of the typical deflagration flame profile captured in the experiment to explore the propagation, growth, and structural changes of the premixed flame under different experimental conditions. Assume that the previous picture before the flame front enters the observation window is $\mathrm{t}=0 \mathrm{~ms}$.
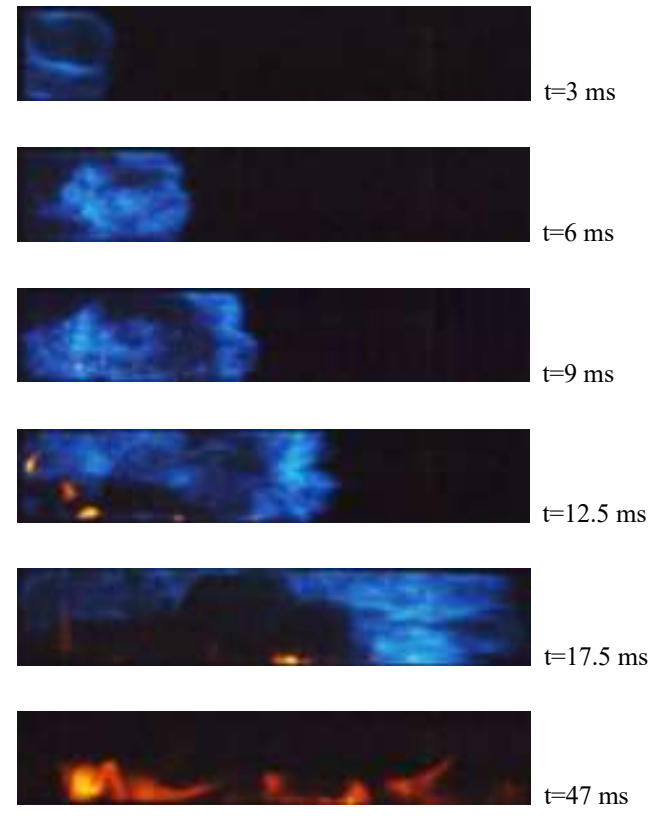
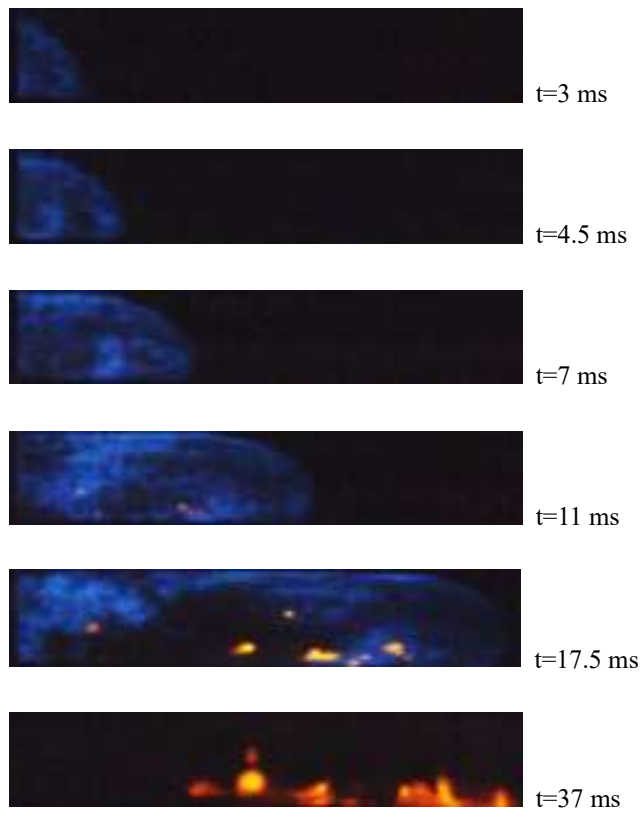
(a) $\Phi=0.9 \quad 0 \% \mathrm{Ar}$

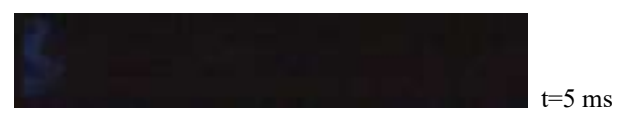

$\mathrm{t}=11.5 \mathrm{~ms}$
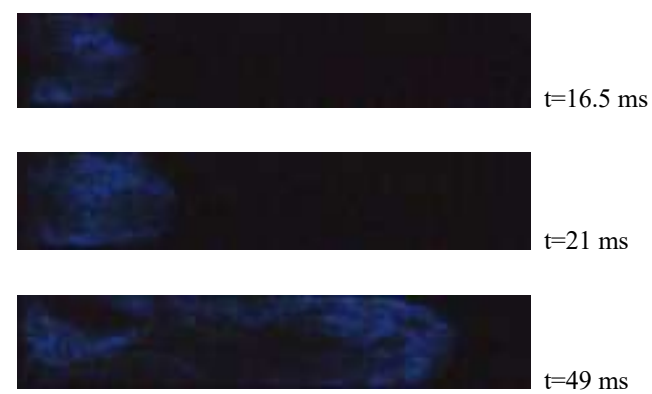

$\mathrm{t}=77.5 \mathrm{~ms}$

(c) $\Phi=0.9 \quad 20 \% \mathrm{Ar}$ (b) $\Phi=0.9 \quad 10 \% \mathrm{Ar}$
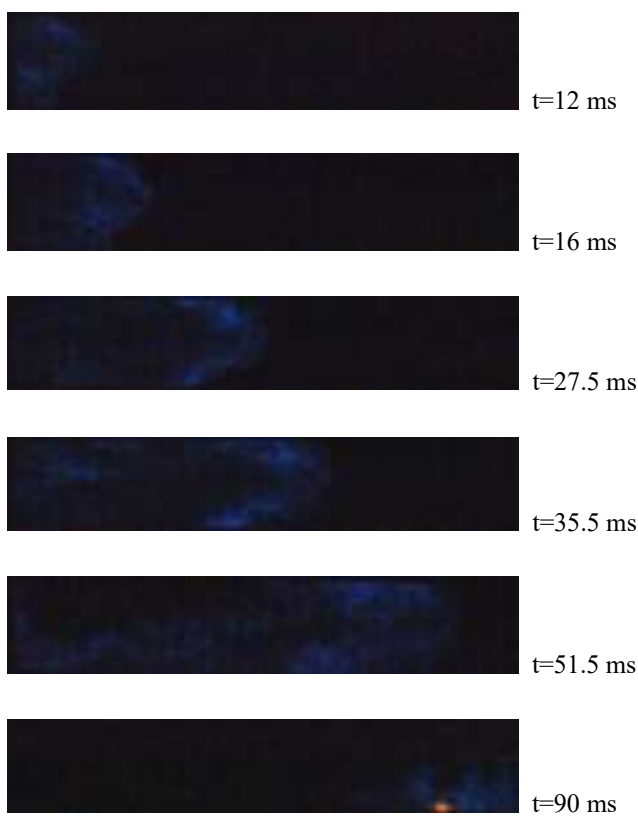

(d) $\Phi=0.9 \quad 30 \% \mathrm{Ar}$

Fig. 3 Flame structure with different proportion of Ar under the condition of $\Phi=0.9$

It can be seen from Fig. 3 that when argon content is 0 , the front of the deflagration flame gradually changes from a "finger" to a flat surface, and then turns inward until it passes through the observation window because of the rise of pressure at $\mathrm{t}=9 \mathrm{~ms}$. With the increase of argon content, the flame front becomes irregular, the propagation time of the "finger-shaped" flame front increases, and the brightness of the premixed flame and the bright yellow flame in the later stage of the combustion gradually decrease.

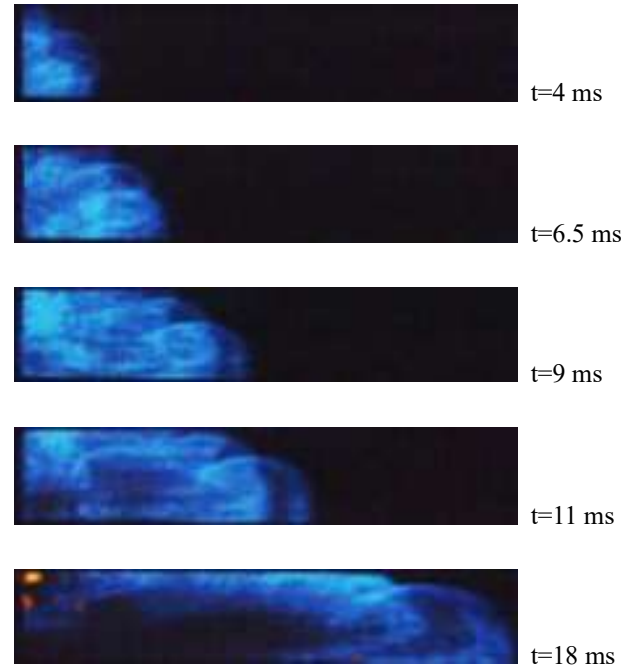


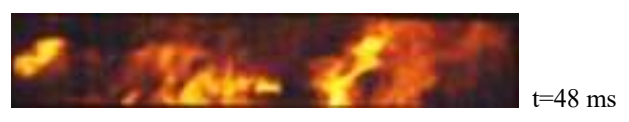

(a) $\Phi=1.0 \quad 0 \% \mathrm{Ar}$
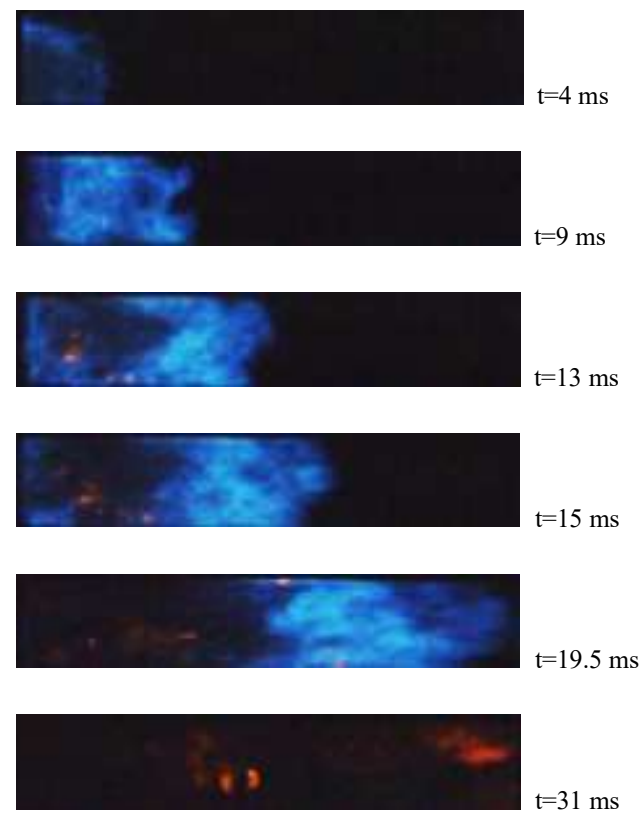

(c) $\Phi=1.0 \quad 20 \% \mathrm{Ar}$

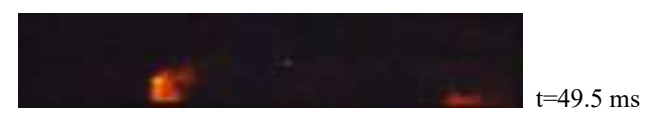

(b) $\Phi=1.0 \quad 10 \% \mathrm{Ar}$
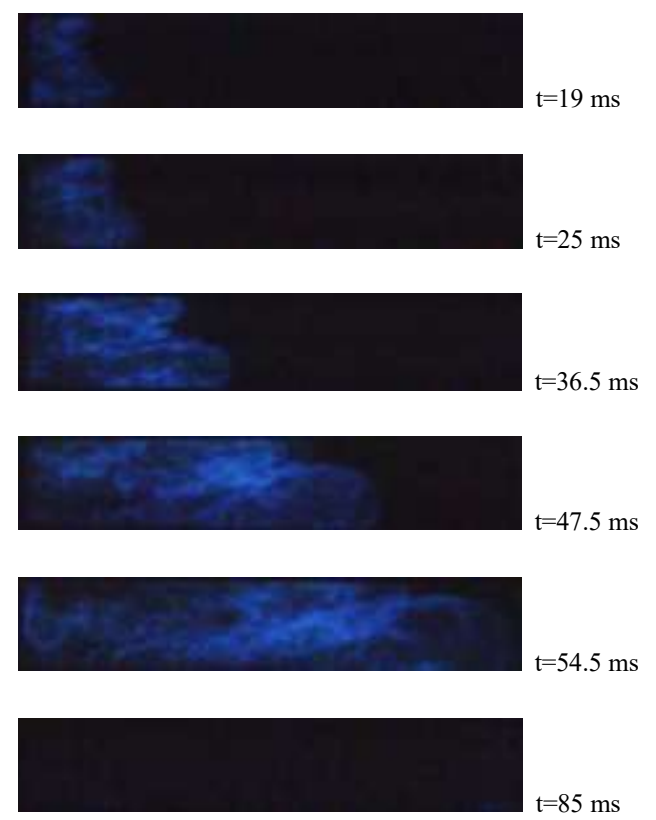

(d) $\Phi=1.0 \quad 30 \% \mathrm{Ar}$

Fig. 4 Flame structure with different proportion of Ar under the condition of $\Phi=1.0$

It can be seen from Fig. 4 that the flame front is mostly a "protruding" structure. With the increase of argon content, the instability of the combustion increase, and the flame brightness decreases, while the brightness of premixed flame under $\Phi=1.0$ condition is higher than that under $\Phi=0.9$ condition under the same ratio of argon added.
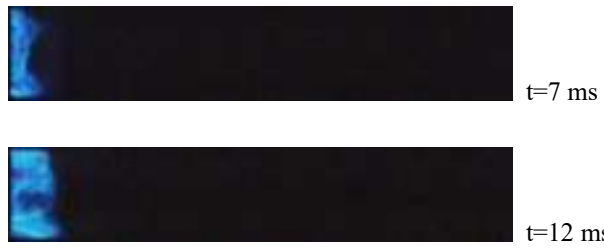

$\mathrm{t}=12 \mathrm{~ms}$


$\mathrm{t}=26.5 \mathrm{~ms}$



$\mathrm{t}=32 \mathrm{~ms}$
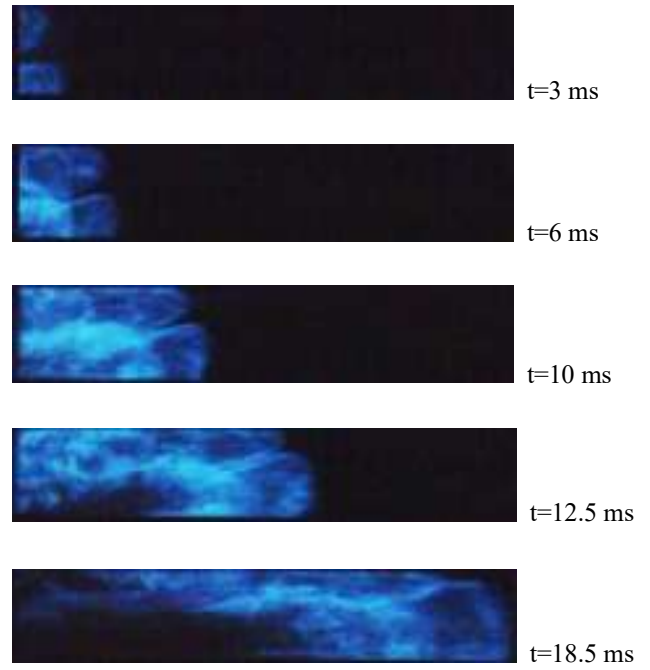


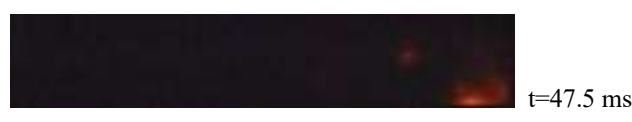

(a) $\Phi=1.1 \quad 0 \% \mathrm{Ar}$
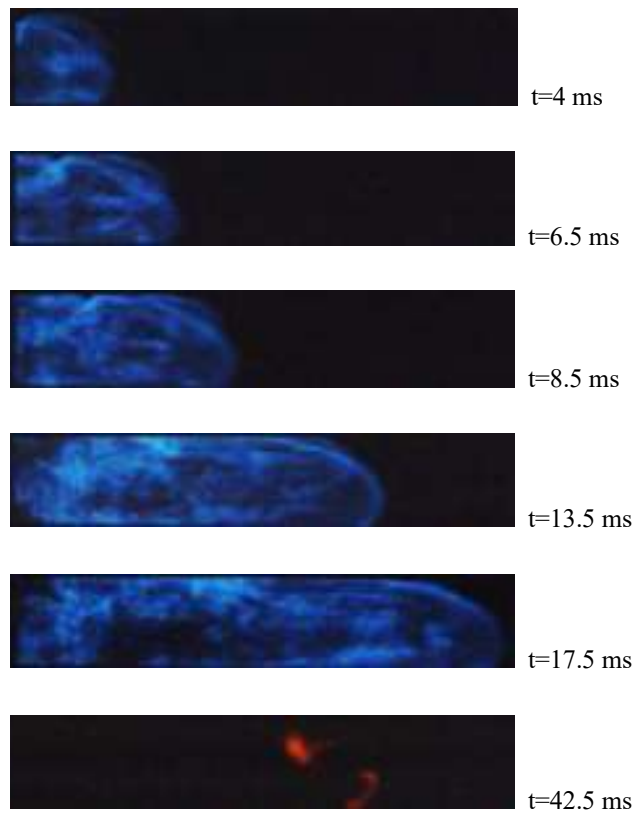

(c) $\Phi=1.1 \quad 20 \% \mathrm{Ar}$

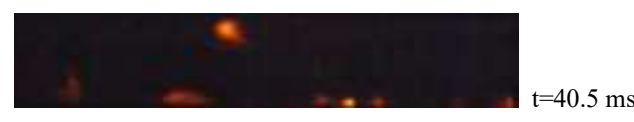

(b) $\Phi=1.1 \quad 10 \% \mathrm{Ar}$
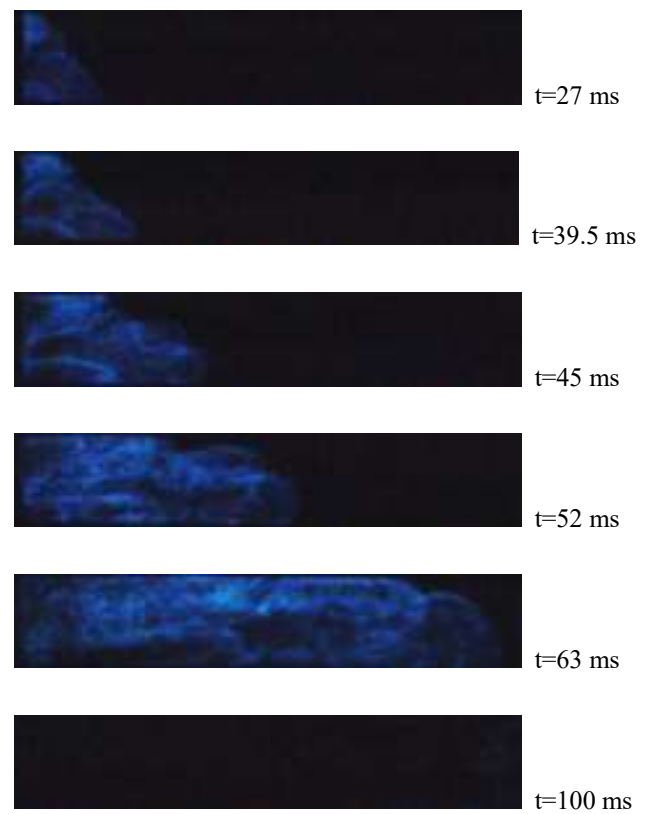

(d) $\Phi=1.1 \quad 30 \% \mathrm{Ar}$

Fig. 5 Flame structure with different proportion of Ar under the condition of $\Phi=1.1$

As shown in Fig. 5, the flame front has been stretched to a certain extent as soon as it enters the observation window when argon content is 0 . The internal concave phenomenon appears with the flame spreads, and then develops into a Tulip flame at $\mathrm{t}=26.5 \mathrm{~ms}$ (Some photos are processed by image enhancement to highlight the Tulip flame characteristics).

Comparing the flame profile photos in Figures 3, 4, and 5, the influence of argon added on the flame structure of methane-air premixed gas at $\Phi=0.9, \Phi=1.0$, and $\Phi=1.1$ decreases in order, which means that the lower the equivalent proportion is, the more obvious the dilution effect of the argon is, and the slower the combustion reactions are. Under the circumstance of same $\Phi$, the flame brightness gradually decreases as the proportion of argon increases.

\subsubsection{Effect of argon on the propagation velocity of methane-air premixed flame}

Figures 6 is graphs of flame propagation velocity in the "near open-end" section of the pipeline with different ratio of Ar. Taking the experimental condition of $\Phi=0.9$ (Fig. 6a) as an example, when argon is not added, the flame propagation velocity curve is relatively smooth, while the velocity curve becomes no longer smooth with the increase of the argon content. The development trend of the flame propagation velocity curve under the circumstance of $\Phi=0.9$ $10 \% \mathrm{Ar}$ is similar to that of $\Phi=0.920 \% \mathrm{Ar}$, which shows a slow rise first and then a large decrease. But the curve under the circumstance of $\Phi=0.930 \% \mathrm{Ar}$ is completely different from the curves mentioned above, showing a development trend of rising first and then falling, and the average flame propagation velocity is the smallest under this experimental 
condition. The specific values are shown in Table 3.

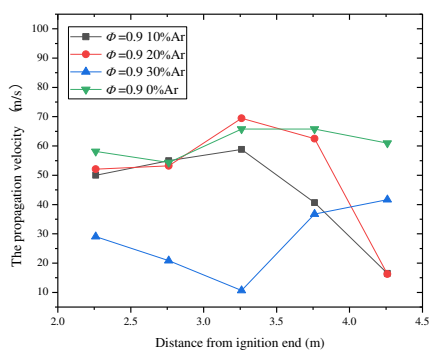

(a) $\Phi=0.9$

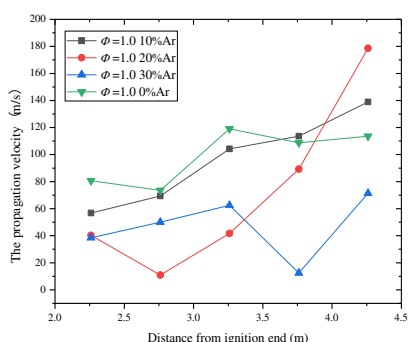

(b) $\Phi=1.0$

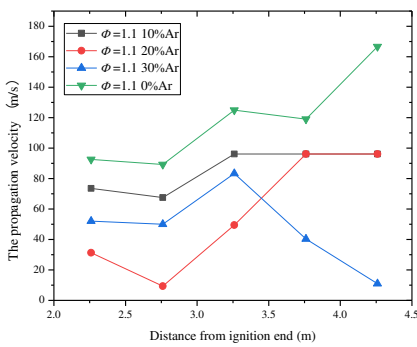

(c) $\Phi=1.1$

Fig. 6 Flame propagation velocity with different ratio of Ar

It can be seen from Fig. $6 \mathrm{~b}$ that under the experimental conditions of $\Phi=1.00 \% \mathrm{Ar}$ and $\Phi=1.010 \% \mathrm{Ar}$, the development trend of the velocity curves are similar, and the average flame propagation velocity under $10 \% \mathrm{Ar}$ condition is slightly lower than that under no argon condition. With the further increase of the argon content, the trend of the speed curve begins to change under the $\Phi=1.020 \%$ Ar experimental condition, and the velocity curve of $\Phi=1.0$ $30 \%$ Ar condition is obviously lower than the other three curves, indicating that the argon content of $30 \%$ has a relatively obvious effect on reducing the flame propagation speed.

Table 3 Research rule of argon suppression on methane-air deflagration flame propagation velocity curve under various experimental conditions

\begin{tabular}{|c|c|c|c|c|c|c|}
\hline \multirow{2}{*}{$\beta_{A r} / \%$} & \multicolumn{3}{|c|}{ Average speed (m/s) } & \multicolumn{3}{|c|}{ Speed decreasing rate $(\%)$} \\
\hline & $\Phi=0.9$ & $\Phi=1.0$ & $\Phi=1.1$ & $\Phi=0.9$ & $\Phi=1.0$ & $\Phi=1.1$ \\
\hline 0 & 61.0 & 99.1 & 118.5 & - & - & - \\
\hline 10 & 44.2 & 96.6 & 85.9 & 27.5 & 2.52 & 27.5 \\
\hline 20 & 50.7 & 72.2 & 56.5 & 16.8 & 27.2 & 52.3 \\
\hline
\end{tabular}




\subsubsection{Effect of argon on flame deflagration pressure of methane-air deflagration}

Figures 7 is time curves of the flame deflagration pressure in the pipeline with different ratio of Ar. It can be seen from the figures that the development trend of the other deflagration pressure-time curves is the same except for the three experimental conditions of $\Phi=0.930 \% \mathrm{Ar}, \Phi=1.030 \% \mathrm{Ar}$, and $\Phi=1.130 \% \mathrm{Ar}$.

In the experiment, two layers of PE film are used as the constraining material at the open end of the pipeline under each experimental condition, and its rupture pressure and material strength, and other parameters are conformed to the following relations 24 :

$$
\Delta P=\frac{\delta \sigma}{d}
$$

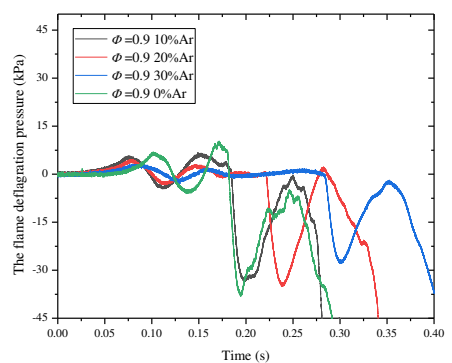

(a) $\Phi=0.9$

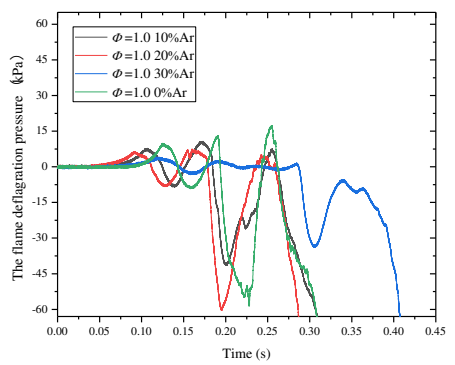

(b) $\Phi=1.0$

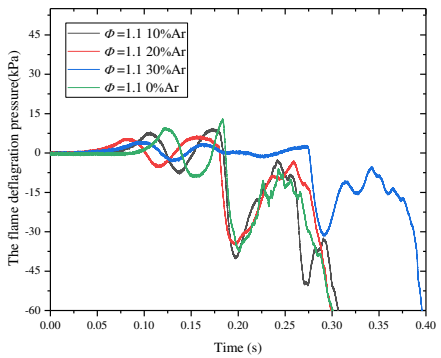

(c) $\Phi=1.1$

Fig. 7 Flame propagation time curve with different ratio of Ar

Here, $\triangle P$ is the pressure difference between the two sides of the diaphragm, $\delta$ is the thickness of the diaphragm, $\sigma$ is the tensile strength, $d$ is diameter of the pressure relief port.

The PE film used in the experiment is $0.02 \mathrm{~mm}$, and its factory-marked tensile strength is about $20 \mathrm{MPa}$. It can be estimated that the rupture pressure of the first layer is $3.3 \mathrm{kPa}$ and the rupture pressure of the second layer is $6.6 \mathrm{kPa}$ according to the above formula. 
High-speed images of a typical film breaking process are shown in Fig. 8. Taking the experimental condition of $\Phi=0.9$ (Fig. 7a) as an example, when the premixed gas in the tube is ignited without breaking the film (Fig. 8b), the pressure in the tube gradually rises and the first pressure peak appears, that is film rupture pressure.

Then the flame turbulence increases, and the deflagration pressure starts to rise due to the change in the flow field of the premixed gas in the tube caused by the film rupture. The flame front reaching the open end of the tube will ignite the unburned premature gas that rushed out of the pipeline after the film was broken. The mixed gas ignites to form an "external explosion" (Fig. 8d), which hinders the release of gas in the pipeline and generates a reverse pressure wave, prompting a rapid increase in deflagration pressure, resulting in a second pressure peak.

When the "external explosion" ends, the combusted gas is quickly released to the outside of the tube, causing negative pressure in the tube and triggering the oscillation of the combustible gas in the tube ${ }^{22}$. This oscillation will cause Taylor instability and further intensify the turbulence degree of the flame in the tube ${ }^{11}$, and finally leads to the appearance of the third pressure peak. The pressure in the tube will eventually return to normal pressure as the combustion reaction ends.

With the further increase of the argon content, the first pressure peak dropping significantly under the $\Phi=0.920 \%$ Ar experimental condition is $4.70 \mathrm{kPa}$, which is not enough to break through the film. Then the flame pressure is relieved from the interface between the film and the tube opening, that is, "edge pressure relief" (Fig. 8g). With the further reaction of the unburned gas in the tube, the pressure rises slightly and the second peak pressure appears, and the final pressure curve remains steady until the flame propagates to the end and burns through the film. After the "burning film" shown in Fig. 8h, the pressure of the flame rushing out of the pipeline drops rapidly. When the content of argon reaches $30 \%$, the first pressure peak value decreases to $2.53 \mathrm{kPa}$. At this time, the pressure is not enough to break through the film of the nozzle, and "edge pressure relief" occurs. The second pressure peak is $1.38 \mathrm{kPa}$ until the flame propagates to the end and burns through the film. And the pressure of the "burning film" flame rushing out of the tube drops rapidly the same as shown in Fig. 8h. The specific values are shown in Table 4.

Table 4 Research rule of argon suppression on methane-air deflagration flame propagation time curve under various experimental conditions

\begin{tabular}{|c|c|c|c|}
\hline$\Phi$ & $\beta_{A r} \%$ & The first pressure peak(kPa) & The second pressure peak $(\mathrm{kPa})$ \\
\hline \multirow{4}{*}{0.9} & 0 & 6.58 & 10.22 \\
\hline & 10 & 5.72 & 6.58 \\
\hline & 20 & 4.70 & 2.43 \\
\hline & 30 & 2.53 & 1.38 \\
\hline 1.0 & 0 & 9.50 & 12.64 \\
\hline
\end{tabular}



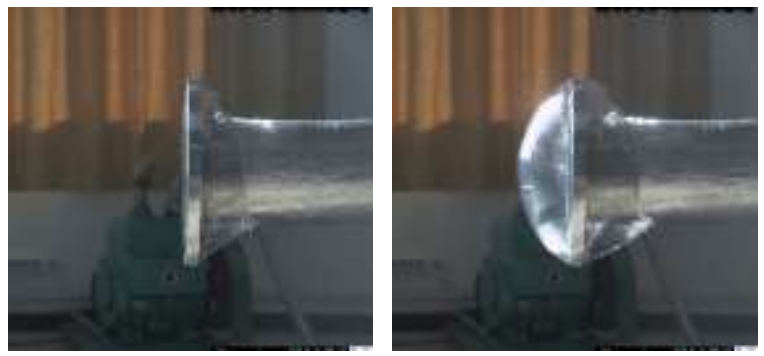

(b) Pressure rise

(a) Ignition started
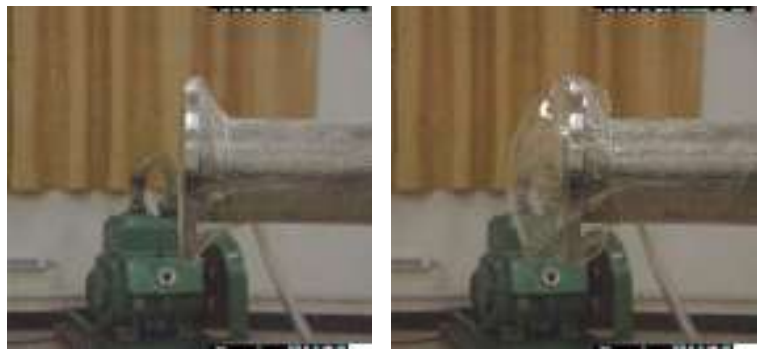

(f) Pressure rise (e) Ignition started (c) Film rupture
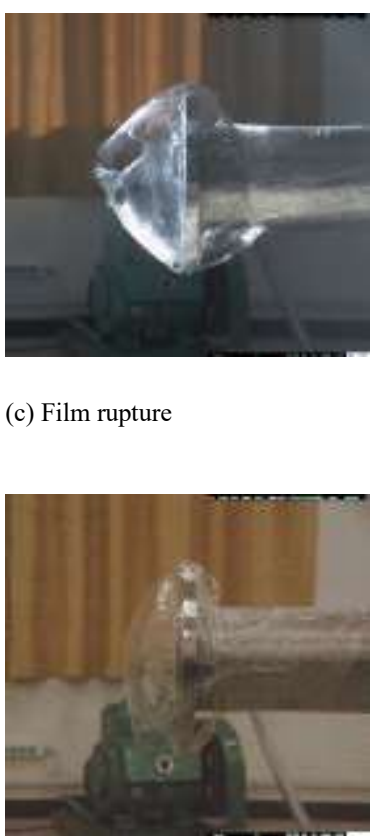

(g) Edge pressure relief

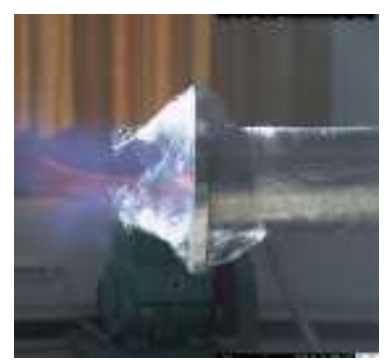

(d) External explosion

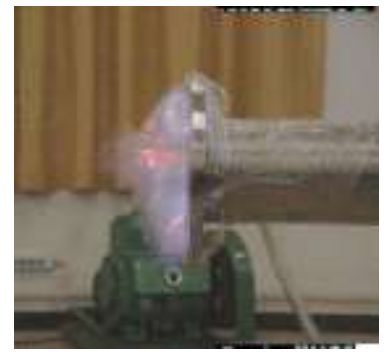

(h) Burning film

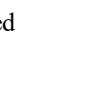


monitored in Fig. 9. It is assumed that the previous photo when the flame front enters the observation window corresponds to $\mathrm{t}=0 \mathrm{~ms}$.

As shown in Fig. 9a, when the flame front enters the observation window, the speed of flame propagation is relatively small at the initial stage, which is approximately laminar combustion. The flame front propagates forward as a spherical surface and then becomes a plane surface at $\mathrm{t}=5 \mathrm{~ms}$, where the flame superficial area is minimum. The flame shows a tensile deformation at $\mathrm{t}=10 \mathrm{~ms}$, and Tulip Flames are gradually formed. It can be seen from Fig. $9 \mathrm{c}$ that a typical Tulip Flame has been formed at $\mathrm{t}=8 \mathrm{~ms}$, and the flame wavefront is further stretched at $\mathrm{t}=9.5 \mathrm{~ms}$, significantly increasing the flame superficial area, intensifying the combustion reaction process, and inducing the transformation of laminar flame to turbulent flame. According to Markstein's model ${ }^{19}$, local propagation velocity of fold flame front can be calculated from the following equation:

$$
v=v_{\mathrm{u}}\left[1+L\left(\frac{1}{R_{\text {flame }}}-\frac{1}{R_{\text {flow }}}\right)\right]
$$

where $v$ and $v_{\mathrm{u}}$ are the local propagation velocity of the fold flame front and the laminar flame propagation, $L$ is the characteristic length, $R_{\text {flame }}$ and $R_{\text {flow }}$ are the radius of curvature at the flame front and the curvature radius of flow field, respectively.

Combining Fig. 9c with the Markstein model, it can be seen that the flame spreads at a laminar velocity as a whole when $R_{\text {flow }}$ and $R_{\text {flame }}$ are the same. But when $R_{\text {flow }}$ and $R_{\text {flame }}$ are different, the local flame velocity will change, which will lead to changes in the flame surface structure. The interaction between the flow change of the flow field in the tube and the flame wavefront is an important reason for the formation of the Tulip Flame ${ }^{20}$.

The flame wavefront under other conditions in Fig. 9 has already been stretched and distorted in a large degree when entering the observation window, and the flame folds have become extremely irregular. The reason for the differentiation of the external characteristics during the flame propagation process might be different equivalence ratio conditions and the unevenness of the tube inwall and other factors ${ }^{21,22}$. The explosion-eliminating chamber mainly acts on the external flame explosion process after the film is broken, and will not have a significant impact on the initial flame structure.
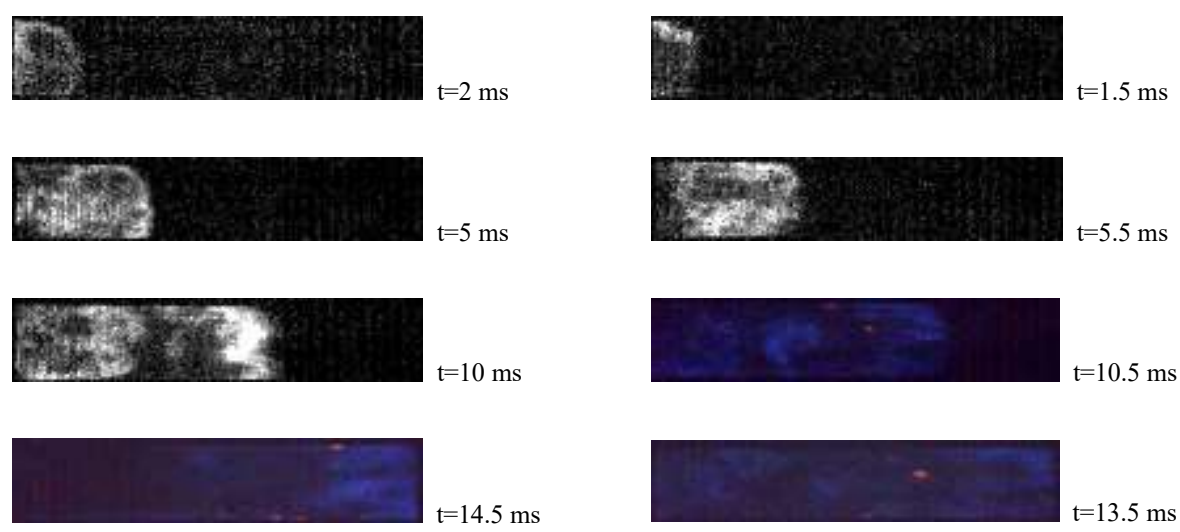

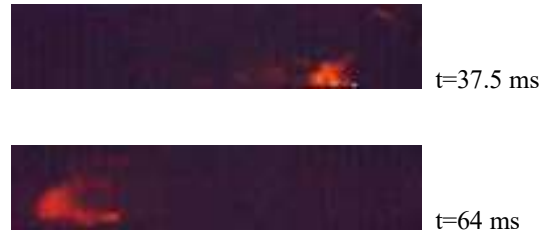

$\mathrm{t}=64 \mathrm{~ms}$

(a) $\Phi=0.9$ without explosion-eliminating chamber
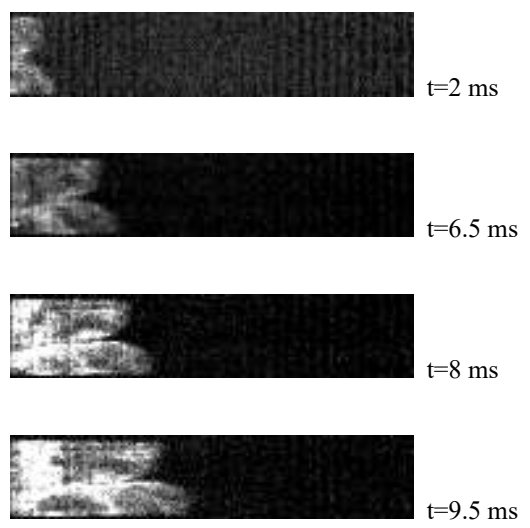

$\mathrm{t}=9.5 \mathrm{~ms}$
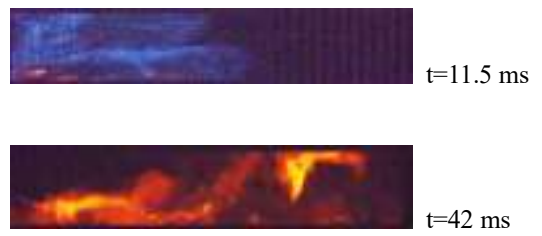

$\mathrm{t}=42 \mathrm{~ms}$

(c) $\Phi=1.0$ without explosion-eliminating chamber
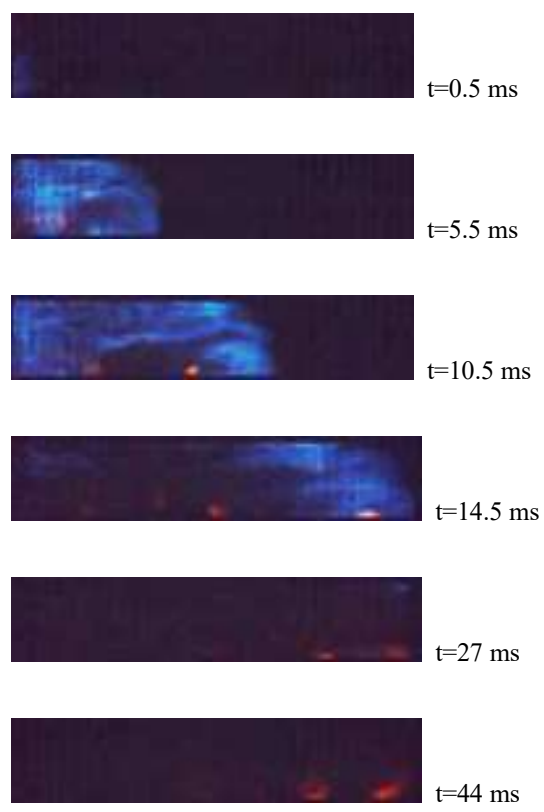

(e) $\Phi=1.1$ without explosion-eliminating chamber
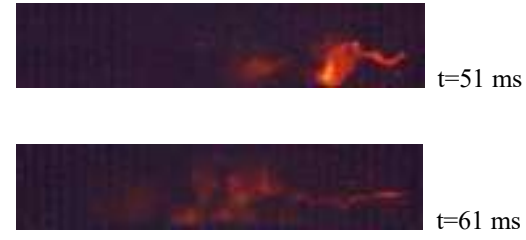

(b) $\Phi=0.9$ with explosion-eliminating chamber
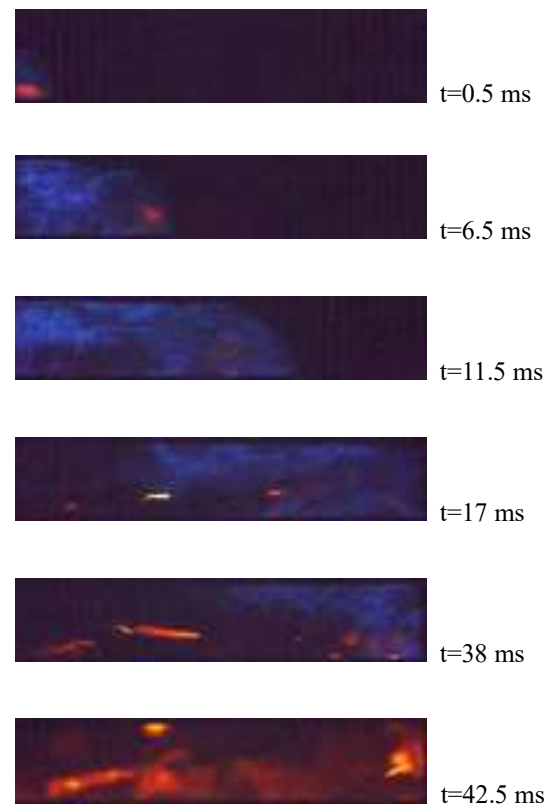

(d) $\Phi=1.0$ with explosion-eliminating chamber
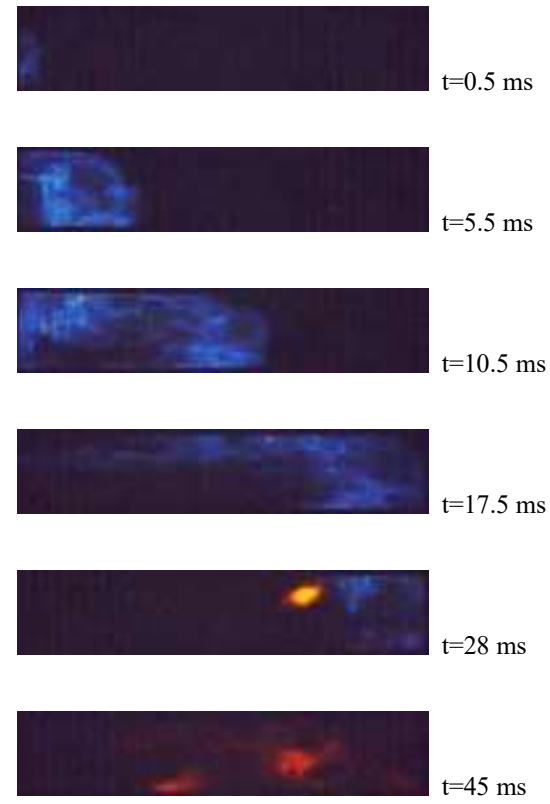

(f) $\Phi=1.1$ with explosion-eliminating chamber 
Fig. 9 High-speed photographs of flame propagation under different experimental conditions

\subsubsection{Effect of the presence or absence of explosion-eliminating chamber on the propagation speed of deflagration flame}

The effect on flame propagation velocity of laying explosion-eliminating chamber is shown in Fig. 10. It can be seen that the trend of the deflagration flame velocity curve under each experimental condition is gradually increasing. This is because more and more combustible gas are involved in the reactions, and flame turbulence has increased as the combustion reaction progresses, which causes the combustion reaction to be more violent. And the speed curves of the laid explosion-eliminating chamber were all located below the curve without the chamber, indicating that the laying of the chamber has a certain limiting effect on flame propagation. The curve of the propagation average velocity of the deflagration flame under all experimental conditions is shown in Fig. 11. The propagation velocity of the deflagration flame is the largest when $\Phi=1.0$. This is because the chemical reaction of the methane-air premixed gas under the theoretical equivalent ratio is the most complete and the combustion reaction is the most violent. The deflagration flame propagation speed under each experimental condition is reduced by $5.7 \%, 12.7 \%$, and $37.0 \%$ respectively compared with the explosion-eliminating chamber conditions, indicating that the laying of the explosion-elimination chamber can significantly attenuate the flame propagation speed. Three explosion suppression rings (annular acoustic absorbing materials) laid in the chamber have a certain energy absorption and buffering effect on the gas release in the pipeline ${ }^{23}$, which will reduce the disturbance of the flow field and then the turbulence of flame propagation in the tube, and ultimately reduce the flame propagation speed in the pipeline ${ }^{20}$. The specific values are shown in Table 5.

Table 5 Research rule of argon suppression on methane-air deflagration flame propagation velocity curve under various experimental conditions

\begin{tabular}{|c|c|c|c|}
\hline$\Phi$ & $\begin{array}{l}\text { With explosion-eliminating } \\
\text { chamber average speed }(\mathrm{m} / \mathrm{s})\end{array}$ & $\begin{array}{c}\text { Without } \\
\text { explosion-eliminating } \\
\text { chamber average speed } \\
(\mathrm{m} / \mathrm{s})\end{array}$ & Speed decreasing rate $(\%)$ \\
\hline 0.9 & 64.14 & 60.51 & 5.7 \\
\hline 1.0 & 131.59 & 114.92 & 12.7 \\
\hline 1.1 & 64.15 & 40.39 & 37.0 \\
\hline
\end{tabular}




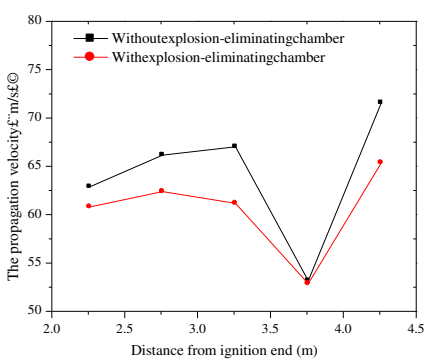

(a) $\Phi=0.9$

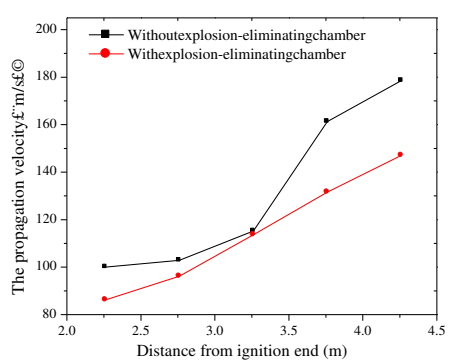

(b) $\Phi=1.0$

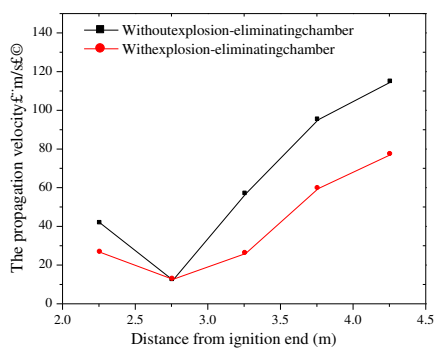

(c) $\Phi=1.1$

Fig. 10 The effect on flame propagation velocity of laying explosion-eliminating chamber

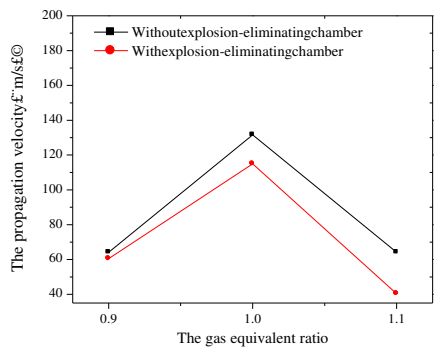

Fig. 11Flame propagation average velocity of different experimental conditions

\subsubsection{Effect of explosion suppression chamber on deflagration pressure}

The effect on flame deflagration pressure of laying explosion-eliminating chamber is shown in Fig. 12. Taking the experimental condition of $\Phi=0.9$ (Fig. 12a) as an example, when the explosion-eliminating chamber is laid, the overall trends of the deflagration pressure change and the physical process of reaching each peak pressure are basically similar to these without the chamber. However, there is a marked decline in the second peak pressure $\mathrm{P}_{\mathrm{b} 2}(9.3 \mathrm{kPa})$ and the third peak pressure $\mathrm{P}_{\mathrm{b} 3}(5.3 \mathrm{kPa})$ due to the laying of the chamber. That is because the deflagration flame at the tube mouth is quenched by the porous structure of the explosion suppression ring inside the explosion-eliminating chamber to a certain extent ${ }^{26}$, and the deflagration flame pressure is significantly reduced by the annular acoustic absorbing material $^{23}$. The pressure generated by the "external explosion" undergoes multiple reflections and attenuation among the three explosion suppression rings in the explosion-eliminating chamber, which reduces the turbulence intensified by the flame in the tube due to the oscillation, and finally reduces the deflagration pressure in the pipeline. In general, the 
second and third pressure peaks will be respectively reduced by about $10 \%$ to $30 \%$ and $50 \%$ to $90 \%$ due to the laying of the explosion-eliminating chamber.

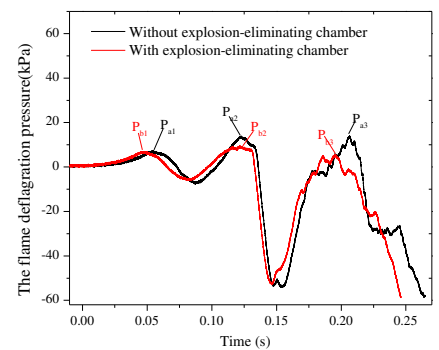

(a) $\Phi=0.9$

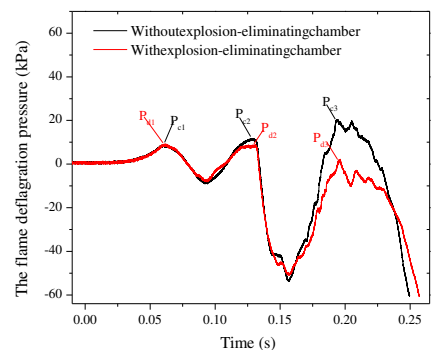

(b) $\Phi=1.0$

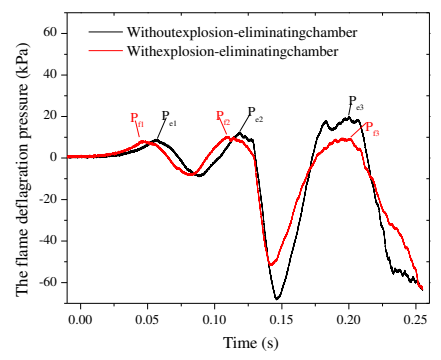

(c) $\Phi=1.1$

Fig. 12 The effect on flame deflagration pressure of laying explosion-eliminating chamber

\subsection{Comparative analysis of the suppression effect of argon gas and explosion-elimination chamber on methane-air deflagration flame}

From the point of view of the suppression effect of argon and the explosion-eliminating chamber on the flame propagation speed, it can be found that adding $10 \% \sim 30 \%$ argon in the methane-air premixed gas with different $\Phi$ reduces the flame propagation speed by $2.52 \% \sim 60.0 \%$, an average reduction of $24.7 \%$, while the laying of the explosion-eliminating chamber at the open end reduces the propagation speed of methane-air deflagration flames with different $\Phi$ by $5.7 \% \sim 37.0 \%$, an average reduction of $18.4 \%$. Thus it can be seen that the inhibitory effect of argon on the flame velocity is better than that of the explosion-eliminating chamber under the experimental conditions.

From the point of view of the suppression effect of argon gas and the explosion-eliminating chamber on the detonation pressure, it can also be found that the suppression effect of argon gas is better than that of laying the explosion-eliminating chamber. The suppression effect of argon on the detonation pressure exists from the beginning of ignition, and with the increase in argon content, the "Rupture pressure" is significantly reduced and "burning film" occurs. It can be seen from the pressure curve that the second and third pressure peaks after the film ruptures are suppressed by laying the explosion-eliminating chamber.

The analysis of the experiment shows that the suppression effect of argon on the methane-air flame propagation 
is better than that of laying an explosion-eliminating chamber. Nevertheless, the time when combustible gas will explode is hard to be predicted in actual production activities, so the inert gas such as argon cannot be injected in time to suppress combustion and explosion. However, explosion-eliminating chamber can be laid in advance at the explosion vents of production equipment, especially when the vents cannot be directly led to the outdoors. From this point of view, explosion-eliminating chamber might be more suitable for practical production than inert gases.

\section{Conclusion}

In the study, the inhibitory effect of argon gases and explosion-eliminating chamber on methane-air deflagration flame were explored by using the flame acceleration pipeline test system. It can be concluded that the inhibitory effect of argon on the methane-air flame propagation is better than that of laying the explosion-eliminating chamber under the experimental conditions, and the following main conclusions were obtained.

(1) The flame propagation can be significantly inhibited by adding argon in methane-air premixed gas, and when the equivalent ratio $\Phi$ is constant, the tensile distortion and instability of the flame front increase with the increase of the mixed argon content, and the brightness of the premixed flame decreases as well.

(2) The average speed of flame propagation and the deflagration pressure can be significantly reduced by adding argon in methane-air premixed gas, especially when the content of argon is $30 \%$. When the equivalence ratio $\Phi$ is

constant, with the increase in the mixed argon content, and the average value of flame propagation speed decreases by $2.52 \% \sim 60.0 \%$. The first and second deflagration pressure peaks are reduced by about $13.1 \% \sim 62 \%$ and $17.7 \% \sim 86.5 \%$ respectively.

(3) The average velocity of flame propagation and the deflagration pressure can be significantly reduced by laying explosion-elimination chambers. The average flame propagation velocity is respectively reduced by $5.7 \%$, $12.7 \%$, and $37.0 \%$ under the condition of $\Phi=0.9, \Phi=1.0$, and $\Phi=1.1$, and the pressure peaks caused by "external explosion" and "flame oscillations intensify turbulence" can be significantly reduced by $10 \% \sim 30 \%$ and $50 \% \sim 90 \%$ respectively.

\section{References}

1. Chen, C. C., Wang, T. C., Liaw, H. J., et al. Nitrogen dilution effect on the flammability limits for hydrocarbons. J. Journal of Hazardous Materials. 166, 880-890 (2009).

2. Chen, C. C., Liaw, H. J., Wang, T. C., et al. Carbon dioxide dilution effect on flammability limits for hydrocarbons. J. Journal of Hazardous Materials. 163, 795-803 (2009) .

3. Degges, M. J., Boyer, J. E., Kuo, K. K., et al. Influence of steam on the flammability limits of premixed natural gas/oxygen/ steam mixtures. J. Chemical Engineering Journal. 165, 633-638 (2010).

4. Kondo, S., Takizawa, K., Takahashi, A., et al. Extended Le Chatelier's formula for carbon dioxide dilution effect on flammability limits. J. Journal of Hazardous Materials. 183, 1-8 (2006).

5. Kondo, S., Takizawa, K., Takahashi, A., et al. Extended Le Chatelier's formula for nitrogen dilution effect on 
the flammability limits. J. Fire Safety Journal. 41, 406-417 (2006).

6. Bundy, M., Hamins, A., Lee, K. Y. Suppression limits of low strain rate non-premixed methane flames. $J$. Combustion and Flame. 133, 299-310 (2003).

7. Benedetto, A. D., Sarli, V. D., Salzano, E. , et al. Explosion behavior of $\mathrm{CH}_{4} / \mathrm{O}_{2} / \mathrm{N}_{2} / \mathrm{CO}_{2}$ and $\mathrm{H}_{2} / \mathrm{O}_{2} / \mathrm{N}_{2} / \mathrm{CO}_{2}$ mixtures. J. International Journal of Hydrogen Energy, 34, 6970-6978 (2009).

8. Mashuga, C. V., Crowl, D. A. Flammability zone prediction using calculated adiabatic flame temperatures. $J$. Process Safety Progress. 18, 127-134 (1999).

9. Shebeko, Y. N., Fan, W., BolodianI, A., et al. An analytical evaluation of flammability limits of gaseous mixtures of combustible-oxidizer-diluent. J. Fire Safety Journal. 37, 549-568 (2002).

10. Vidal, M., Wong, W, Rogers, W. J., et al. Evaluation of lower flammability limits of fuel-air-diluent mixtures using calculated adiabatic flame temperatures. J. Journal of Hazardous Materials. 130, 21-27 (2006).

11. Cooper, M. G., Fairweather, M., Tite, J. P. On the mechanisms of pressure generation in vented explosions. $J$. Combust Flame. 65, 1-14 (1986).

12. Bauwens, C. R., Chaffee, J., Dorofeev, S. Effect of ignition location, vent size, and obstacles on vented explosion overpressures in propane-air mixtures. J.Combust Science and Technology. 182, 1915-1932 (2010).

13. Ponizy, B., Leyer, J. C. Flame dynamics in a vented vessel connected to a duct. Mechanism of vessel-duct interaction. J. Combust and Flame. 116, 259-271 (1999).

14. Ferrara, G, Benedetto, A . D., Salzano E., et al. CFD analysis of gas explosions vented through relief pipes. $J$. Journal of Hazardous Materials. 137, 654-665 (2006).

15. Solberg, D. M., Pappasj, A., Skramstad, E. Observations of flame instabilities in large scale vented gas explosions. J. Symposium on Combustion. 18, 1607-1614 (1981).

16. Bradley, D., Mitcheson, A. The venting of gaseous explosions in spherical vessels, I-Theory. J. Combustion and Flame. 32, 237-255 (1978).

17. Kasmani, R. M., Andrews, G. E. Phylaktou, H. N. Experimental study on vented gas explosion in a cylindrical vessel with a vent duct. J. Process Safety and Environmental Protection. 91, 245-252 (2013).

18. Alexiou, A., Andrews, G. E., Phylaktou, H. A comparison between end-vented and side-vented gas explosions in largeL/D vessels. J. Process Safety and Environmental Protection. 75, 9-13 (1997).

19. Markstein, G. H. Nonsteady flame propagation, New York, Pergamum Press, 1964.

20. Chen, X. X., SUN, J. H., Yao, L. Y., et al. Characteristics of fine structure during tulip flame forming. $J$. Journal of Combustion Science and Technology. 4, 350-354 (2008).

21. Yu, M., Yang, X., Zheng, K., et al. Experimental study of premixed syngas/air flame propagation in a half-open duct. J. Fuel. 225, 192-202 (2018). 
22. Wang, Q., Shen, Z. W., Guo, Z. R., et al. Analysis on propagation characteristics of premixed methane-air flame in an half-open tube based on high-speed video camera. J. Explosive Materials. 5, 18-22 (2013).

23. Nian, W. M., Zhou, K. Y., Xia, C. J., et al. The experimental study of strength attenuation of gaseous etonation wave through acoustic absorbing walled channel. J. Fire Safety Science. 12, 84-89 (2003).

24. Wang, Q., Guo, Z. R., Shen, Z. W., et al. Study on deflagration pressure distribution of combustible gas in PMMA square tube. J. Initiators \& Pyrotechnics. 5, 18-21 (2012).

25. Guo, J., Wang, C., Li, Q., et al. Effect of the vent burst pressure on explosion venting of rich methane-air mixtures in a cylindrical vessel. J. Journal of Loss Prevention in the Process Industries. 40, 82-88 (2015).

26. Nie, B., He, X., Zhang, R., et al. The roles of foam ceramics in suppression of gas explosion overpressure and quenching of flame propagation. J. Journal of Hazardous Materials. 192, 741-747 (2011).

\section{Acknowledgements}

This study was supported by National Natural Science Foundation of China (No. 11872002) and Doctoral Introduction Foundation of AUST (No.13210003), the authors expressed their sincere gratitude here.

\section{Author contributions}

All authors contributed and reviewed the manuscript.

\section{Competing interests}

Te authors declare no competing interests. 